\title{
On Some Variants of Euclidean K-Supplier
}

\author{
Euiwoong Lee* Viswanath Nagarajan ${ }^{\dagger} \quad$ Lily Wang $^{\dagger}$
}

December 6, 2021

\begin{abstract}
The $k$-Supplier problem is an important location problem that has been actively studied in both general and Euclidean metrics. Many of its variants have also been studied, primarily on general metrics. We study two variants of $k$-Supplier, namely Priority $k$-Supplier and $k$-Supplier with Outliers, in Euclidean metrics. We obtain $(1+\sqrt{3})$-approximation algorithms for both variants, which are the first improvements over the previously-known factor-3 approximation (that is known to be best-possible for general metrics). We also study the Matroid Supplier problem on Euclidean metrics, and show that it cannot be approximated to a factor better than 3 (assuming $P \neq N P$ ); so the Euclidean metric offers no improvement in this case.
\end{abstract}

\section{Introduction}

In the $k$-Supplier problem, the input consists of a set of suppliers $I$ and a set of clients $J$ contained in some metric space $(I \cup J, d)$, and $k \in \mathbb{N}$. The goal is to choose a subset $C \subseteq I$ of $k$ suppliers to $\operatorname{minimize} \max _{v \in J} d(v, C)$ where $d(v, C):=\min _{u \in C} d(v, u)$. A basic problem in the large and well-studied class of location problems, $k$-Supplier has various applications in operations research including choosing sites for opening plants, placing servers in a network, and clustering data. An important special case of $k$-Supplier is $k$-Center where the set of clients $J$ is equal to the set of suppliers $I$.

The approximability of $k$-Supplier and $k$-Center on general metric spaces is well understood. A 2 -approximation for $k$-Center and 3-approximation for $k$-Supplier follow from the work of Gonzalez Gon85] and Hochbaum and Shmoys [HS85, HS86. Simple reductions from Vertex Cover show that these approximation ratios are tight assuming $\mathbf{P} \neq \mathbf{N P}$.

However, the approximability of $k$-Supplier and $k$-Center on Euclidean metrics (which is a practically important special case) is still open. Feder and Greene [FG88] showed that it is NPhard to approximate $k$-Supplier and $k$-Center better than $\sqrt{7} \approx 2.65$ and $\sqrt{3} \approx 1.73$ respectively. While it is still open whether one can obtain a $(2-\epsilon)$-approximation for $k$-Center for some constant $\epsilon>0$, Nagarajan et al. [NSS20] obtained a $(1+\sqrt{3}) \approx 2.73$ approximation algorithm for Euclidean $k$-Supplier.

Motivated by various practical needs, many variants of $k$-Supplier and $k$-Center also have been proposed and studied in the literature. In the Priority $k$-Supplier problem, the clients are additionally weighted with a priority function $p: V \rightarrow \mathbb{R}_{+}$. Given a set of chosen suppliers $C \subseteq I$, the

\footnotetext{
${ }^{*}$ Computer Science and Engineering, University of Michigan.

${ }^{\dagger}$ Industrial and Operations Engineering, University of Michigan. Research supported in part by NSF grants CMMI-1940766 and CCF-2006778.
} 
objective function is now $\max _{v \in J} p(v) d(v, C)$. This problem naturally models the scenario where each client has a different "speed". Plesnik [Ple87] gave a 3-approximation algorithm for Prioirty $k$-Supplier, matching the approximability of the basic version.

Another variant is $k$-Supplier with Outliers where the input additionally contains a bound $\ell \in \mathbb{N}$ and the goal is to choose $k$ suppliers $C \subseteq I$ and $\ell$ outliers $O \subseteq J$ to $\operatorname{minimize} \max _{v \in J \backslash O} d(v, C)$. This problem was introduced by Charikar et al. CKMN01. Recently, Chakrabarty et al. [CGK20] obtained a 3-approximation algorithm for this problem, again matching the approximability of the basic $k$-Supplier problem. Yet another variant is the Matroid Supplier problem: instead of a cardinality bound on the chosen suppliers, the set $C$ of chosen suppliers is required to be independent in some matroid. Chen et al. [CLLW13] obtained a 3-approximation algorithm for this problem as well.

Results and Techniques. To the best of our knowledge, the study of the above $k$-Supplier variants has been limited to general metrics. In this paper, we study these problems in Euclidean metrics. Our first result is the following:

Theorem 1. There is an $(1+\sqrt{3}) \approx 2.73$-approximation algorithm for Euclidean Priority $k$ Supplier.

This is based on a relation to the minimum edge-cover problem, as in [NSS20. However, the graph for the edge-cover instance is constructed differently: we need to select "representative" clients (that correspond to nodes in the graph) in decreasing order of their priorities.

Our second and main technical result is the following:

Theorem 2. There is an $(1+\sqrt{3}) \approx 2.73$-approximation algorithm for Euclidean $k$-Supplier with Outliers.

This requires a linear-program (LP) in conjunction with the relation to edge-cover. Moreover, we do not know how to solve the resulting LP in polynomial time. Instead, we use a "round or cut" approach that is built atop the ellipsoid algorithm, and in each step it either finds an approximate solution or a violated LP constraint. We note that round-or-cut has been used recently to address some other $k$-Supplier problems [CN19, but the focus there was on general metrics and dealing with complex constraints on the suppliers. In contrast, our goal is to exploit the Euclidean metric to improve the approximation ratio (beyond 3). Another important step in proving Theorem 2 is an integrality property for the edge-cover polytope with a special type of cardinality constraint; this result might also be of some independent interest.

Finally, we show that not all natural variants of $k$-Supplier are strictly easier in Euclidean metrics. In particular, we consider the Matroid Supplier problem where there is a matroid constraint

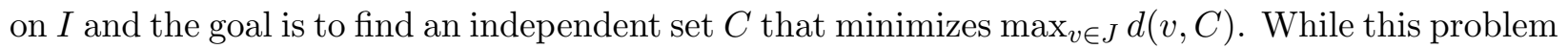
admits a 3-approximation algorithm in general metrics [CN19], we prove the following theorem that Euclidean spaces do not strictly improve the approximation ratio.

Theorem 3. For any constant $\epsilon>0$, it is NP-hard to approximate Euclidean Matroid Supplier within a factor of $(3-\epsilon)$.

Other Related Work. Apart from $k$-Supplier/ $k$-Center, such variants have also been studied for $k$-Median (where the objective is to minimize the sum of connection costs). In particular, 
there are constant-factor approximation algorithms for $k$-Median with outliers [Che08, KLS18, and Matroid Median [KKN ${ }^{+}$15, Swa16]. Moreover, there is an extensive literature on obtaining better approximation ratios (and runtime) for $k$-Median on Euclidean metrics, see e.g., [KR07, HPM04.

\section{$2 \quad k$-Supplier with Priorities}

Given a set of suppliers $I$ and clients $J$, where clients have a priority function $p: J \rightarrow \mathbb{R}_{+}$, the goal is to choose $k$ suppliers to minimize the maximum "priority weighted distance" over all clients. That is, we want to find

$$
\min _{\substack{C \subseteq I \\|C| \leq k}} \max _{v \in J} p(v) \cdot d(v, C) .
$$

For a given set of suppliers $C$, the priority distance of any client $v \in J$ is $p(v) \cdot d(v, C)$.

Assuming optimal value of 1 . As is common for min-max optimization problems (see e.g., [HS85]), we assume that the algorithm knows the optimal value $B$. Then, the algorithm either finds a solution of objective at most $\alpha \cdot B$ (where $\alpha$ is the approximation ratio), or proves that the optimal value is more than $B$. As there are only a polynomial number of choices for $B$, we can try each one. Finally, by scaling all distances by $B$, we can assume that the optimal value is 1 .

Our algorithm is similar to that in NSS20 for the basic $k$-Supplier. This involves constructing a graph with some clients $S \subseteq J$ as nodes and suppliers as edges, and finding the minimum edgecover in this graph. The key difference is that we need to include clients into the node-set $S$ in decreasing order of priorities. See Algorithm 1 for details.

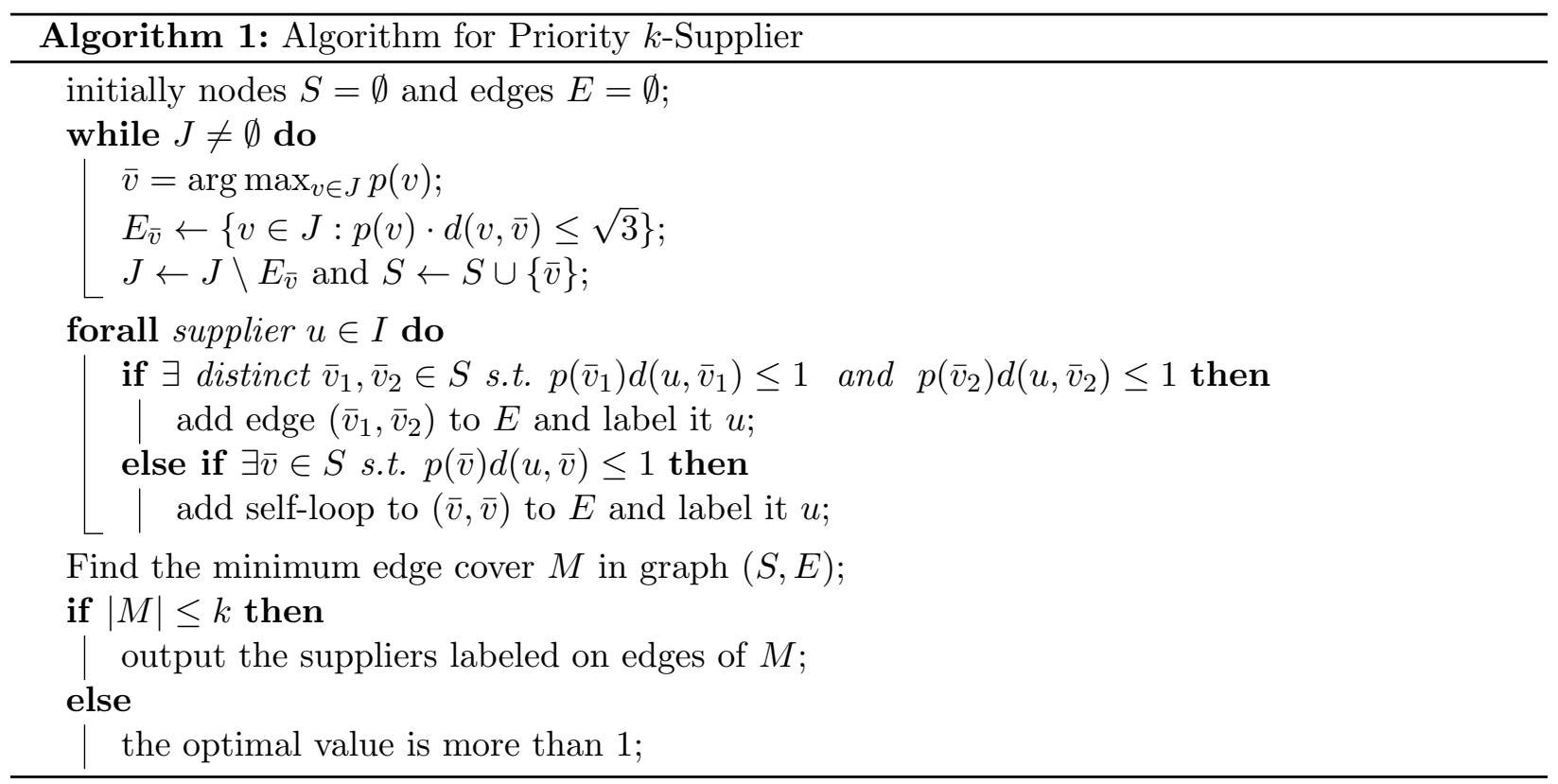

For the analysis, we will show that if the optimal value is at most 1 , the algorithm returns solution $M$ with objective at most $1+\sqrt{3}$. Henceforth, we assume that the optimal value is at most 1 . 


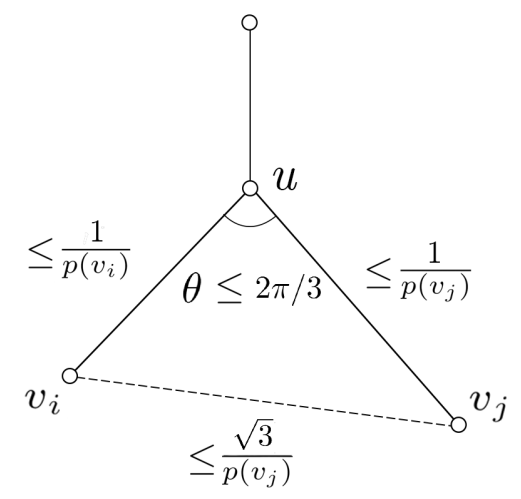

Figure 1: Illustration of Lemma 2,

Lemma 1. Each client $v \in J$ is within priority-distance $(1+\sqrt{3})$ from some supplier in $M$.

Proof. Consider any $v \in J$ : it must lie in $E_{\bar{v}}$ for some "selected" client $\bar{v} \in S$. Note that $\bar{v}$ must be covered by some edge in $M$, say labelled by supplier $u$. Then,

$$
p(v) d(v, u) \leq p(v) d(v, \bar{v})+p(v) d(\bar{v}, u) \leq p(v) d(v, \bar{v})+p(\bar{v}) d(\bar{v}, u) \leq \sqrt{3}+1 .
$$

The second inequality uses the fact that at the point when $\bar{v}$ was added to $S$, client $v$ was also in $J$ : so $p(\bar{v}) \geq p(v)$. The third inequality is by definition of $E_{\bar{v}}$ and edges $E$.

Lemma 2. No supplier can serve more than two clients of $S$ within priority-distance 1.

Proof. Suppose for a contradiction that for supplier $u \in I$ and clients $v_{1}, v_{2}, v_{3} \in S$ are within priority-distance 1 from $u$. Then, we have $p\left(v_{i}\right) d\left(v_{i}, u\right) \leq 1$ for $i=1,2,3$. There is at least one pair of $v_{i}, v_{j}$ such that the angle $\theta=\angle v_{i} u v_{j} \leq 2 \pi / 3$. See Figure 1. Suppose without loss of generality that $p\left(v_{i}\right) \geq p\left(v_{j}\right)$, so that $v_{i}$ is added to $S$ before $v_{j}$. By the cosine law,

$$
\begin{aligned}
d\left(v_{i}, v_{j}\right) & =\sqrt{d\left(v_{i}, u\right)^{2}+d\left(v_{j}, u\right)^{2}-2 \cdot d\left(v_{i}, u\right) \cdot d\left(v_{j}, u\right) \cdot \cos \theta} \\
& \leq \sqrt{\frac{1}{p\left(v_{i}\right)^{2}}+\frac{1}{p\left(v_{j}\right)^{2}}+\frac{1}{p\left(v_{i}\right) p\left(v_{j}\right)}} \leq \sqrt{\frac{3}{p\left(v_{j}\right)^{2}}}=\frac{\sqrt{3}}{p\left(v_{j}\right)}
\end{aligned}
$$

It follows that

$$
p\left(v_{j}\right) d\left(v_{j}, v_{i}\right) \leq p\left(v_{j}\right) \frac{\sqrt{3}}{p\left(v_{j}\right)}=\sqrt{3} .
$$

Therefore, $v_{j}$ should have been in $E_{v_{i}}$ and can not be in $S$, a contradiction.

Lemma 3. The minimum edge cover $M$ satisfies $|M| \leq k$.

Proof. Let $M^{*} \subseteq I$ be the optimal set of suppliers. Note that $M^{*}$ covers each client within priority distance 1. Moreover, by Lemma 2, each supplier can cover at most two clients of $S$ within priority distance 1. In other words, taking the edges corresponding to the suppliers $M^{*}$ in graph $(S, E)$, we get an edge cover. Therefore, the minimum edge cover $M$ has size at most $\left|M^{*}\right|=k$.

Combining the lemmas above, we obtain Theorem 1. 


\section{$3 \quad k$-Suppliers with Outliers}

Here, we are given a set of suppliers $I$ and clients $J$ along with bounds $k$ on the number of chosen suppliers and $\ell$ on the number of outlier clients. As mentioned earlier, we assume that the optimal value is 1 , and aim to find a solution with objective at most $1+\sqrt{3}$. This would prove Theorem 2 .

We start with a natural LP relaxation where decision variables $y_{i}$ correspond to selecting suppliers and $z_{j}$ correspond to choosing outlier clients.

$$
\begin{aligned}
& \sum_{i \in I} y_{i} \leq k \\
& z_{j}+\sum_{i \sim j} y_{i} \geq 1 \quad \forall j \in J \\
& \sum_{j \in J} z_{j} \leq \ell \\
& 0 \leq z, y \leq 1
\end{aligned}
$$

Above, $i \sim j$ denotes client $j$ being within unit distance from supplier $i$, i.e., supplier $i$ can serve client $j$. While these constraints suffice to obtain a 3-approximation algorithm (even on general metrics), we need to add stronger constraints for the improved $1+\sqrt{3}$ approximation ratio.

Define a subset of clients $S \subseteq J$ to be well-separated if all pairwise distances in $S$ are greater than $\sqrt{3}$, i.e., $d\left(j, j^{\prime}\right)>\sqrt{3}$ for every $j, j^{\prime} \in S$. Also, for any set of clients $S$, we will denote the set of suppliers which can serve at least one client in $S$ by $f(S) \subseteq I$. The stronger constraints we want to add are the following:

$$
z(S)+y(f(S)) \geq\lceil|S| / 2\rceil \quad \forall S \subseteq J \text { well-separated. }
$$

Above, we use the shorthand $z(S):=\sum_{j \in S} z_{j}$ and $y(f(S)):=\sum_{i \in f(S)} y_{i}$.

We now show that these constraints are valid for any (integral) solution to $k$-Supplier with Outliers. Consider any well-separated set $S$. Note that no supplier can serve more than two clients in $S$ : this follows from Lemma 2 with all priorities being 1 (or Lemma 1 in [NSS20]). Hence, a total of at least $\lceil|S| / 2\rceil$ suppliers from $f(S)$ or outliers in $S$ are needed to "cover" the clients in $S$.

Our final LP relaxation, referred to as the "Master LP" consists of constraints (1)-(4) and (5). There are an exponential number of well-separated constraints, and we are not aware of a separation oracle for these. So, this LP is difficult to solve directly. Instead, we will use a roundor-cut approach that either (i) finds a solution of objective at most $1+\sqrt{3}$, or (ii) proves that the Master LP is infeasible. Note that case (ii) also implies that the optimal value of the $k$-Supplier with Outliers problem is more than 1 . So this would suffice to prove Theorem 2.

We are now ready to describe the algorithm, which relies on the ellipsoid algorithm with separation-oracles. We will maintain a candidate solution $(y, z)$ for the Master-LP, and an ellipsoid $\mathcal{F}$ that is guaranteed to contain Master-LP.

In each iteration below, we either (i) find an approximate solution to $k$-Supplier with Outliers, or (ii) identify a violated constraint for the Master-LP, which is used to update our solution $(y, z)$ and the ellipsoid $\mathcal{F}$. Formally, we repeat the following steps.

1. If $(y, z)$ violates any of the (polynomially many) constraints (1)-(4), then update solution $(y, z)$ and ellipsoid $\mathcal{F}$ based on the violated constraint. Continue to the next iteration. 
2. Let nodes $A=\emptyset, B=J$.

3. Order clients by outlier values from the LP solution: $z_{1} \leq z_{2} \leq \ldots \leq z_{n}$, where $n=|J|$.

4. While $B \neq \emptyset$ do:

- Let $j \in B$ with the client with lowest $z_{j}$.

- Take all clients in $B$ within distance $\sqrt{3}$ of $j$ (including itself) and assign them to $R_{j}$.

- Remove $R_{j}$ from the set $B$.

- Let $a(j)=\left|R_{j}\right|$ denote the number of clients assigned to $j$.

- Add node $j$ to $A$.

5. Construct a graph $G$ with nodes $A$ and the following edges. For each supplier $i$ :

- If there are two distinct clients $j_{1}, j_{2} \in A$ within distance 1 from $i$, add edge $\left(j_{1}, j_{2}\right)$ labelled by $i$.

- Otherwise, if there is just one client $j \in A$ within distance 1 from $i$, add self-loop $(j, j)$ labelled by $i$.

Let $E$ be the set of all edges added above. Furthermore, add a distinct set $L$ of self-loops at each vertex $j \in A$ : the loop at $j$ represents making $j$ an outlier. All edges of $E$ have weight 0 . Each loop $(j, j)$ in $L$ has weight $a(j)$.

6. Check whether $(y, z)$ satisfies the following constraints:

$$
z(S)+y(f(S)) \geq\lceil|S| / 2\rceil \quad \forall S \subseteq A .
$$

These constraints exactly specify the edge cover polytope of graph $G=(A, E \dot{\cup} L)$ and can be efficiently checked [Sch03].

7. If $(y, z)$ violates $(6)$ for some $S \subseteq A$, then:

- Update solution $(y, z)$ and the ellipsoid $\mathcal{F}$ based on the constraint for $S$. (Note that the constraint for $S$ appears in (5) of the Master-LP as $S \subseteq A$ is well-separated.)

- Continue to the next iteration.

8. If $(y, z)$ satisfies (6), apply Theorem 4 below to obtain a solution $M$ to min-weight edgecover on graph $G$ with a cardinality constraint on $E$. Output the suppliers in $M \cap E$ as the approximate solution, and stop.

Assuming that the algorithm never stops in step 8, the standard analysis for the ellipsoid algorithm (see e.g., GLS88]) implies that we can terminate after a polynomial number of iterations and conclude that the Master-LP is infeasible. Therefore, the overall algorithm is guaranteed to run in polynomial time. Moreover, we either return some solution $M$ (in step 8) or prove that the Master-LP is infeasible. In the analysis below, we will show that the solution $M$ obtained in step 8 is a $1+\sqrt{3}$ approximation for $k$-Supplier with Outliers. 
Edge cover with a cardinality constraint. Consider a graph $G$ on nodes $A$ and edges $E^{\prime}=$ $E \cup \dot{\cup} L$, where $L$ only contains self-loops. (Edges in $E$ can be 2-edges or self-loops.) Note that we use the same notation as for the graph constructed in step 5 of the above algorithm. Each edge $e \in E^{\prime}$ has a weight $w_{e}$. We are interested in solving the minimum weight edge-cover problem on $G$ subject to a cardinality constraint of $k$ on $E$. That is, we want a min-weight edge cover $M \subseteq E^{\prime}$ where $|M \cap E| \leq k$. Note that the cardinality constraint does not include all edges $E^{\prime}$, but only those in set $E$. We will show that this problem can be solved in polynomial time using the natural LP relaxation.

Consider the following linear program $L P_{E C C}$ for the above edge-cover problem with a cardinality constraint. Recall that the edges are $E^{\prime}=E \dot{\cup} L$. We use decision variables $y \in \mathbb{R}^{E}$ for the edges in $E$ and $z \in \mathbb{R}^{L}$ for the remaining edges $L$.

$$
\begin{array}{lr}
\sum_{e \in E} y_{e} \leq k & \left(L P_{E C C}\right) \\
z(S)+y(f(S)) \geq\lceil|S| / 2\rceil & \forall S \subseteq A \\
z, y \geq 0 &
\end{array}
$$

Theorem 4. $L P_{E C C}$ is integral. Moreover, there is a polynomial time algorithm for the min-weight edge-cover problem with a cardinality constraint.

We defer the proof of this theorem to Section 3.1 .

Completing the proof of Theorem 2, We now use Theorem 4 to show that the solution $M$ found in step 8 of our algorithm is a feasible solution to $k$-Supplier with Outliers of objective at most $1+\sqrt{3}$.

When the algorithm reaches step 8, observe that all constraints in (6) are satisfied by the current solution $(y, z)$. Moreover, by step 1, all the basic constraints (1)-(4) are also satisfied. It follows that this solution $(y, z)$ is also feasible for $L P_{E C C}$. By definition of the edge-weights in the edge-cover instance, the weight objective of this solution is:

$$
\sum_{e \in E} w_{e} \cdot y_{e}+\sum_{(j, j) \in L} w_{(j, j)} \cdot z_{j}=\sum_{j \in A} a(j) \cdot z_{j}=\sum_{j \in A}\left|R_{j}\right| \cdot z_{j} \leq \sum_{j \in A} \sum_{j^{\prime} \in R_{j}} z_{j^{\prime}} \leq \sum_{j^{\prime} \in J} z_{j} \leq \ell .
$$

The first inequality uses the fact that we select clients into $A$ in increasing order of $z$-values: so $z_{j} \leq z_{j^{\prime}}$ for all $j^{\prime} \in R_{j}$. The second inequality uses that $\left\{R_{j}: j \in A\right\}$ are disjoint. The last inequality uses constraint (3). Therefore, the integral solution $M$ to $L P_{E C C}$ (found by Theorem 4) has weight $\sum_{e \in M} w_{e}=\sum_{(j, j) \in M \cap L} a(j) \leq \ell$. Let $A^{\prime} \subseteq A$ denote the clients/nodes in graph $G$ that are covered by the edges $M \cap E$. Note that every client in $A^{\prime}$ is within distance 1 from some supplier of $M \cap E$. Hence, every client in $\cup_{j \in A^{\prime}} R_{j}$ is within distance $\sqrt{3}+1$ from $M \cap E$. Moreover, $M \cap L$ must contain the loops at each of the clients $A \backslash A^{\prime}$. It then follows that $\sum_{j \in A \backslash A^{\prime}}\left|R_{j}\right| \leq \sum_{(j, j) \in M \cap L} a(j) \leq \ell$. We set $O=\cup_{j \in A \backslash A^{\prime}} R_{j}$ to be the outlier clients. From the above discussion, it is clear that each non-outlier client is within distance $\sqrt{3}+1$ from $M \cap E$ and the number of outliers $|O| \leq \ell$. Finally, $|M \cap E| \leq k$ because of the cardinality constraint. It now follows that $M \cap E$ is a feasible solution to $k$-Supplier with Outliers of objective at most $1+\sqrt{3}$. 


\subsection{Proof of Theorem 4}

We note that if the set $L=\emptyset$ (i.e., the cardinality constraint involves all edges) then $L P_{E C C}$ is known to be integral: see the discussion in page 464 of [Sch03]. However, this does not directly imply Theorem 4. Moreover, the following example shows that Theorem 4 is not true for a cardinality constraint on an arbitrary edge subset. Hence, our proof below relies crucially on the fact that $L$ only contains self-loops.

Example: suppose graph $G$ is a 4 -cycle with edges $a, b, c, d$ in that order. The cardinality constraint is imposed on $E=\{a, c\}$, with a bound of $k=1$. Note that any integral solution to $L P_{E C C}$ must be of the form $(0, \alpha, 0, \beta),(1, \alpha, 0, \beta)$ or $(0, \alpha, 1, \beta)$, where $\alpha, \beta \in \mathbb{Z}_{\geq 1}$. It can be checked directly that the solution $\left(\frac{1}{2}, \frac{1}{2}, \frac{1}{2}, \frac{1}{2}\right)$ cannot be written as a convex combination of integer solutions, which shows that $L P_{E C C}$ is not integral for this instance.

Recall that the set of edges is $E^{\prime}=E \dot{\cup} L, y \in \mathbb{R}^{E}$, and $z \in \mathbb{R}^{L}$, where the set $L$ only contains self-loops. ( $E$ may contain self-loops too.) For any multi-subset $S \subseteq E^{\prime}$ of edges, we use $\mathbf{1}(S) \in \mathbb{Z}^{E^{\prime}}$ to denote the vector of multiplicities. Before proving Theorem 4 , we show the following key lemma.

Lemma 4. Consider any feasible solution $(y, z)$ for $L P_{E C C}$. There is a collection $\left\{J_{i}\right\}_{i=1}^{r}$ of integral solutions (i.e, edge covers that satisfy the cardinality constraint) and convex multipliers $\left\{\lambda_{i}\right\}_{i=1}^{r}$ such that $(y, z) \geq \sum_{i=1}^{r} \lambda_{i} \cdot \mathbf{1}\left(J_{i}\right)$.

Proof. Fix any fractional solution $(y, z)$ to $L P_{E C C}$. Clearly, this is also feasible to the basic edgecover LP (without the cardinality constraint). By integrality of the edge-cover LP (Theorem 27.3 of [Sch03]), it follows that $(y, z)$ dominates a convex combination of integral edge-covers. Let $(y, z) \geq \sum_{a=1}^{r} \lambda_{a} \cdot \mathbf{1}\left(J_{a}\right)$ denote such a convex combination where the $J_{a}$ are integral edge-covers

and the $\lambda_{a}$ are convex multipliers. Over all such possible convex combinations, choose the one which produces the least "variance" as measured by

$$
\sum_{a=1}^{r} \lambda_{a} \cdot \max \left(0,\left|E \cap J_{a}\right|-k\right) .
$$

We can assume (without loss of generality) that each integral edge-cover $J_{a}$ is minimal. Indeed, if $J_{a}$ is not minimal, we can replace it by a minimal edge-cover $\bar{J}_{a} \subsetneq J_{a}$ : the variance of the resulting convex combination can only decrease.

If the variance is 0 then we must have $\left|E \cap J_{a}\right| \leq k$ for every $a$, which implies that each $J_{a}$ is an integral solution to $L P_{E C C}$. In this case, the lemma is trivially true.

We now suppose (for a contradiction) that the variance is positive. As the variance is positive, we have some $i \in[r]$ with $\left|E \cap J_{i}\right| \geq k+1$ by integrality. As $(y, z)$ satisfies the cardinality constraint, we have $\sum_{a=1}^{r} \lambda_{a}\left|E \cap J_{a}\right| \leq \sum_{e \in E} y_{e} \leq k$. Therefore, there is some $\ell \in[r]$ with $\left|E \cap J_{\ell}\right| \leq k-1$ (again by integrality).

Let $C=E \cap J_{i}$ and $C_{o}=L \cap J_{i}$. Note that both $C$ and $C_{o}$ are sets (not multisets) because of minimality of $J_{i}$. Likewise, let $D=E \cap J_{\ell}$ and $D_{o}=L \cap J_{\ell}$.

We now convert edge-cover $J_{i}=C \dot{\cup} C_{o}$ into a perfect matching (with loops) as follows.

1. Let $\bar{C} \subseteq C$ be any maximal matching using only 2-edges. 


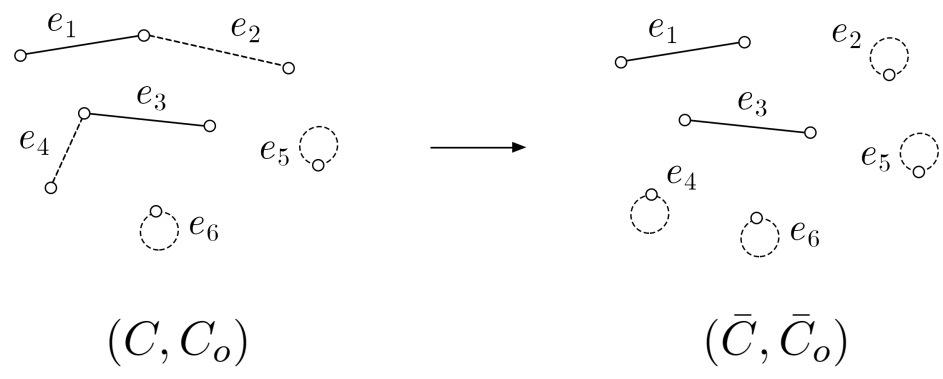

Figure 2: Converting edge-cover to a perfect matching.

2. Then, for any other edge $e=(u, v) \in\left(C \cup C_{o}\right) \backslash \bar{C}$, if one of its nodes (say $u$ ) is incident to the matching $M$ then we modify $e$ into the self-loop $(v, v)$; otherwise edge $e$ remains unchanged. Note that such an edge $e$ cannot have both nodes $u, v$ incident to matching $M$, by minimality of edge-cover $C \cup C_{o}$.

Let $\bar{C}_{o}$ denote all edges created in step 2 above. Note that $\bar{C} \cup \bar{C}_{o}$ is a perfect matching: each node has exactly one edge (either 2-edge or self-loop) incident to it. Note also that there is a 1-to-1 correspondence between the edge-covers $J_{i}=C \cup C_{o}$ and $\bar{C} \cup \bar{C}_{o}$. See Figure 2 for an example.

We apply the same procedure to modify edge-cover $J_{\ell}=D \dot{\cup} D_{o}$ into $\left(\bar{D}, \bar{D}_{o}\right)$. We now have two graphs, each of which is a perfect matching (with self-loops). Let $\mathcal{G}$ denote the disjoint union $\bar{C} \dot{\cup} \bar{C}_{o} \dot{\cup} \bar{D} \dot{\cup} \bar{D}_{o}$ of all these edges. Note that each connected component in $\mathcal{G}$ is either an even cycle (with 2-edges) or a path with self-loops at both ends.

Assign a value of 1 (resp. -1) to all edges in $\bar{C} \dot{\cup} \bar{C}_{o}$ (resp. $\bar{D} \dot{\cup} \bar{D}_{o}$ ) that correspond to $E$-edges. All the other edges (corresponding to $L$-edges) are assigned value 0 . Note that every 2-edge has value +1 or -1 . Over the entire graph, the total value is positive as

$$
\left|\left(\bar{C} \dot{\cup} \bar{C}_{o}\right) \cap E\right|=|C|>|D|=\left|\left(\bar{D} \dot{\cup} \bar{D}_{o}\right) \cap E\right| .
$$

So, there is some component $H$ in $\mathcal{G}$ with positive total value. Note that component $H$ cannot be a cycle: any cycle is even and hence has value 0 . So $H$ is a path with self-loops at both ends. (The path may also be empty, in which case we have a node with two self-loops.) Moreover, the 2-edges on the path have alternating positive or negative value. The self-loops at the end of path $H$ have either 0 value or the opposite sign as the 2-edge they're incident to. Hence, component $H$ has total value $-1,0$, or 1 . Since it has positive value, it must have value exactly 1 . We now define two new edge-covers: $X$ (resp. $Y$ ) consists of the edges from $J_{i}$ (resp. $J_{\ell}$ ) in all components except $H$, and edges from $J_{\ell}$ (resp. $J_{i}$ ) in component $H$. Note that $X$ and $Y$ are indeed edge covers. Moreover, $|X \cap E|=\left|J_{i} \cap E\right|-1=|C|-1$ and $|Y \cap E|=\left|J_{\ell} \cap E\right|+1=|D|+1$.

We now construct a new convex combination that has smaller variance, which leads to a contradiction. Recall the edge-covers $\left\{J_{a}\right\}_{a=1}^{r}$ in the original convex combination. Let $J_{r+1}=X$ and $J_{r+2}=Y$ be the two new edge-covers. Let $\epsilon=\min \left\{\lambda_{i}, \lambda_{\ell}\right\}>0$. The convex multipliers are now:

$$
\lambda_{a}^{\prime}= \begin{cases}\lambda_{a}-\epsilon & \text { if } a=i, \ell \\ \epsilon & \text { if } a=r+1, r+2, \quad \forall a \in[r+2] . \\ \lambda_{a} & \text { otherwise }\end{cases}
$$



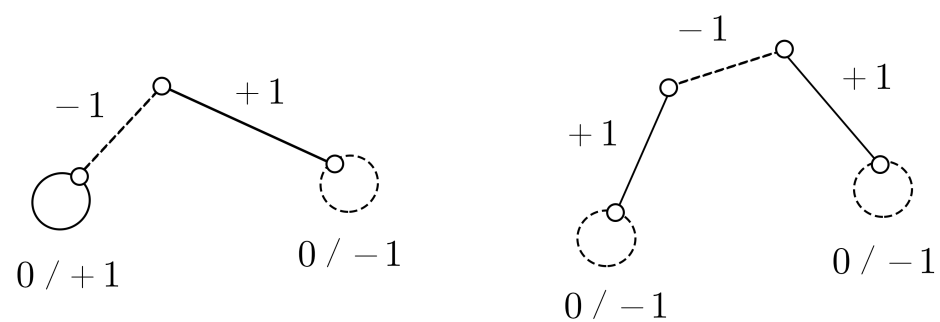

Figure 3: Cases for a component $H$ with positive value

Clearly, $\sum_{a=1}^{r+2} \lambda_{a}^{\prime} \cdot \mathbf{1}\left(J_{a}\right)=\sum_{a=1}^{r} \lambda_{a} \cdot \mathbf{1}\left(J_{a}\right) \leq(y, z)$. We now bound the increase in variance:

$$
\begin{aligned}
& \sum_{a=1}^{r}\left(\lambda_{a}^{\prime}-\lambda_{a}\right) \max \left(0,\left|E \cap J_{a}\right|-k\right)+\epsilon \cdot \max (0,|C|-1-k)+\epsilon \cdot \max (0,|D|+1-k) \\
= & -\epsilon \cdot \max (0,|C|-k)-\epsilon \cdot \max (0,|D|-k)+\epsilon \cdot \max (0,|C|-1-k)+\epsilon \cdot \max (0,|D|+1-k) \\
\leq & -\epsilon
\end{aligned}
$$

where the last inequality uses the fact that $|C|-1-k \geq 0 \geq|D|+1-k$. As $\epsilon>0$, this contradicts the choice of the original convex combination (of minimum variance). This completes the proof.

Continued Proof of Theorem 4. We first prove the integrality of $L P_{E C C}$. Given any fractional solution $(y, z)$ to $L P_{E C C}$, Lemma 4 implies $(y, z) \geq \sum_{i=1}^{r} \lambda_{i} \cdot \mathbf{1}\left(J_{i}\right)$ for some convex combination of integral solutions. We now show that we can ensure equality, i.e., $(y, z)$ is equal to a convex combination of integral solutions. Clearly, this would prove that $L P_{E C C}$ is integral.

We can write $(y, z)=\sum_{i=1}^{r} \lambda_{i} \cdot \mathbf{1}\left(J_{i}\right)+\left(y^{\prime}, z^{\prime}\right)$ for some $y^{\prime} \in \mathbb{R}^{E}, z^{\prime} \in \mathbb{R}^{L}$ with $y^{\prime}, z^{\prime} \geq 0$. Note that $k \geq y(E)=\sum_{i=1}^{r} \lambda_{i}\left|J_{i} \cap E\right|+y^{\prime}(E)$. So, if $y^{\prime}(E)>0$, there exists $i \in[r]$ such that $\left|J_{i} \cap E\right|<k$. Choose an edge $e \in E$ such that $y_{e}^{\prime}>0$. We now perform one of the following modifications that maintains $(y, z)=\sum_{i=1}^{r} \lambda_{i} \cdot \mathbf{1}\left(J_{i}\right)+\left(y^{\prime}, z^{\prime}\right)$ and $y^{\prime}, z^{\prime} \geq 0$ while strictly decreasing $y^{\prime}(E)$.

1. If $\lambda_{i}>y_{e}^{\prime}$, let $\lambda_{i} \leftarrow \lambda_{i}-y_{e}^{\prime}$ and create a new index $r+1$ such that $\lambda_{r+1}=y_{e}^{\prime}$ and $J_{r+1}=$ $J_{i} \dot{\cup}\{e\}$. Let $r \leftarrow r+1$ and $y_{e}^{\prime}=0$.

2. If $\lambda_{i} \leq y_{e}^{\prime}$, let $J_{i} \leftarrow J_{i} \dot{\cup}\{e\}$ and $y_{e}^{\prime} \leftarrow y_{e}^{\prime}-\lambda_{i}$.

The above step 1 strictly decreases the support of $y^{\prime}$, so cannot be done more than $|E|$ times. Between two consecutive applications of step 1's, each application of step 2 strictly increases the size of one $J_{i}$ : so it can be done at most $r k$ times. (And $r$ increases by at most one for each application of step 1.) Therefore, the above procedure can be repeatedly applied and finished in finite time so that $y^{\prime}(E)=0$ at the end. The same procedure can be applied for $z^{\prime}$ as well, which is even easier because we do not have the cardinality constraint for $L$. At the end, we have $(y, z)=\sum_{i=1}^{r} \lambda_{i} \cdot \mathbf{1}\left(J_{i}\right)$ where each $J_{i}$ is an integral edge cover that satisfies the cardinality constraint. We note that these edge-covers $J_{i}$ may be multisets (and not minimal edge covers).

To obtain a polynomial time algorithm for min-weight edge-cover with a cardinality constraint, we first solve $L P_{E C C}$ optimally using the ellipsoid algorithm. This can be done because there is an efficient separation oracle for the edge-cover LP. The resulting solution $(y, z)$ may not be an 
extreme point of $L P_{E C C}$ (and hence not integral). However, we can apply a standard polynomialtime method for converting an arbitrary LP solution into an extreme point solution (assuming a separation oracle for the constraints); see e.g., Lemma 3.3 in Jai01. Hence, we can find an optimal extreme point solution $\left(y^{*}, z^{*}\right)$ to $L P_{E C C}$ in polynomial time. By integrality of $L P_{E C C},\left(y^{*}, z^{*}\right)$ is an integral optimal solution.

\section{Hardness for Matroid Supplier}

We now consider the Euclidean Matroid Supplier problem. Its input consists of $I \cup J \subseteq \mathbb{R}^{s}$ and a matroid $\mathcal{I}$ on ground set $I$, and the goal is to find an independent set $C \in \mathcal{I}$ that minimizes $\min _{j \in J} d(j, C)$, where $d$ denotes the Euclidean distance. We prove that this problem is $(3-\epsilon)$-hard to approximate for any constant $\epsilon>0$, proving Theorem 3 .

We reduce from the NP-hard 1-in-3-SAT problem [Sch78]. This involves $n$ binary variables and $m$ clauses, each consisting of three literals (of any variable or its negation). The goal is to decide whether there is an assignment where exactly one literal is true in each clause.

Suppose that we have a $(3-\epsilon)$-approximation algorithm for Euclidean Matroid Supplier (for any $\epsilon>0)$. Define $c:=\frac{2 \pi}{\cos ^{-1}\left(1-\frac{\epsilon}{2}\right)}$. Given any instance $\mathcal{H}$ of 1 -in-3-SAT, we generate an instance $\mathcal{E}$ of Euclidean Matroid Supplier as follows. Let the variables in $\mathcal{H}$ be $x_{1}, \ldots, x_{n}$, and suppose it has $m$ clauses. Let $d$ be an integer with $d \geq \max \left(\frac{c+1}{4}, m\right)$. In $\mathcal{E}$, we create $n$ cycles embedded as regular $4 d$-gons of unit side length, with each cycle $C_{i}$ representing variable $x_{i}$. The cycles are placed far apart so that no vertex is within distance 3 of a vertex from a different cycle. For each cycle $C_{i}$, we label its vertices alternatively as clients and suppliers. Moreover, the suppliers on cycle $C_{i}$ are alternatively labeled as $x_{i}$ or $\neg x_{i}$. More precisely, if the vertices on $C_{i}$ are numbered $j=1,2, \ldots, 4 d$ then we label the vertices as follows:

$$
f(j)= \begin{cases}x_{i} \text { (supplier) } & \text { if } j \equiv 0 \bmod 4 \\ \neg x_{i} \text { (supplier) } & \text { if } j \equiv 2 \bmod 4 \\ c_{i j} \text { (client) } & \text { otherwise. }\end{cases}
$$

Note that the number of suppliers in each cycle is $2 d$, leading to $2 n d$ suppliers in total. Let $I$ denote the set of all suppliers. Now we construct a partition matroid over $I$ in the following way. For each clause $k \in[m]$, say involving variables $x_{i_{1}}, x_{i_{2}}, x_{i_{3}}$, part $P_{k} \subseteq I$ consists of one supplier each from cycles $C_{i_{1}}, C_{i_{2}}, C_{i_{3}}$, where we take a supplier labeled $x_{i_{j}}$ (resp. $\neg x_{i_{j}}$ ) if the clause uses $x_{i_{j}}$ (resp. $\neg x_{i_{j}}$ ). See Figure 4 for an example. We ensure that each supplier is in at most one part: that is possible because each cycle contains $d \geq m$ suppliers of each label. Finally, we gather all suppliers not in any part $P_{k}$ into another part $P_{0}=I \backslash\left(\cup_{k=1}^{m} P_{k}\right)$. The partition matroid is required to pick at most one supplier from each part $\left\{P_{k}\right\}_{k=1}^{m}$ and at most $d n-m$ suppliers from part $P_{0}$.

Yes case. Suppose that the 1-in-3-SAT instance $\mathcal{H}$ is satisfiable by some assignment $a=\left\{a_{i}\right\}_{i=1}^{n}$ of variables. Consider the Matroid Supplier solution $S$ that selects from each cycle $C_{i}$ all the $x_{i}$ (resp. $\neg x_{i}$ ) suppliers if $a_{i}=$ true (resp. $a_{i}=$ false). The total number of selected suppliers $|S|=d n$. Note that each client is within distance one from some supplier in $S$. Moreover, for each clause $k \in[m]$, exactly one literal of this clause is true in assignment $a$ : this implies that $\left|S \cap P_{k}\right|=1$. It follows that a total of $m$ suppliers are selected from $\cup_{k=1}^{m} P_{k}$, which means $\left|S \cap P_{0}\right|=n d-m$. Hence, $S$ satisfies the partition matroid constraint. So, the optimal value of instance $\mathcal{E}$ is at most 1. 


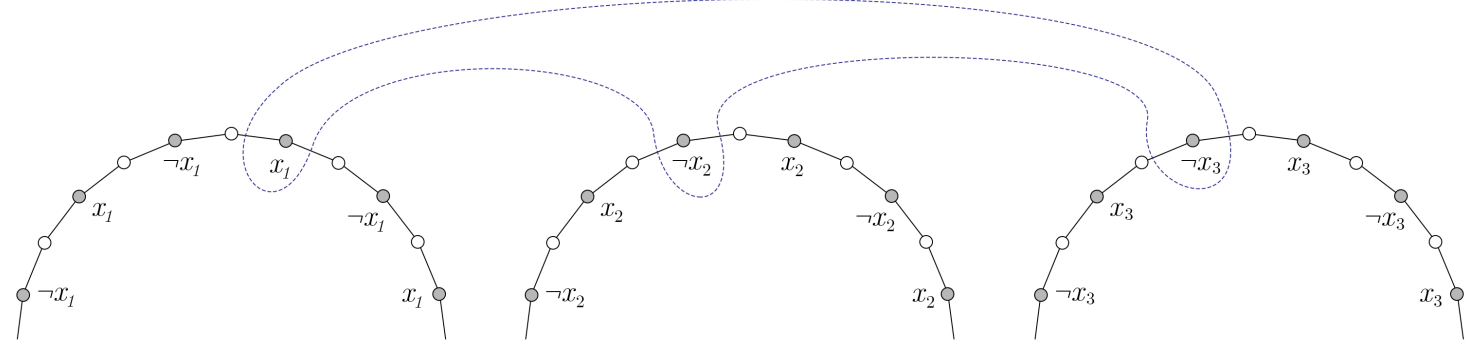

Figure 4: Example of the part corresponding to clause $x_{1} \vee \neg x_{2} \vee \neg x_{3}$.

No case. Suppose that $S^{\prime}$ is a solution to Matroid Supplier of objective at most $3-\epsilon$. Note that the distance between any client and supplier is either 1 or at least $1+2 \cos \left(\pi-\frac{4 d-2}{4 d} \pi\right)>$ $1+2 \cos \left(\frac{2}{c} \pi\right)=3-\epsilon$. So the objective value of solution $S^{\prime}$ must be one.

Claim 1. Consider any solution $S^{\prime}$ to $\mathcal{E}$ with objective 1 . For each $i \in[n], S^{\prime}$ contains either all the $x_{i}$ suppliers or all the $\neg x_{i}$ suppliers in cycle $C_{i}$. Moreover, $\left|S^{\prime} \cap P_{k}\right|=1$ for all $k \in[m]$.

Proof. By the matroid constraint it is clear that $\left|S^{\prime}\right| \leq d n$. Note that each supplier is at unit distance from at most 2 clients, and each cycle has $2 d$ clients. Therefore, solution $S^{\prime}$ must contain at least $d$ suppliers in each cycle $C_{i}$. As there are $n$ cycles, we must have $\left|S^{\prime}\right|=d n$, and the first statement follows. To see the second statement, note that the only way we can have $\left|S^{\prime}\right|=d n$ is to pick exactly one supplier from each $\left\{P_{k}\right\}_{k=1}^{m}$.

Now, consider the assignment $a_{i}^{\prime}=$ true if $S^{\prime}$ contains all the $x_{i}$-suppliers in cycle $C_{i}$, and $a_{i}^{\prime}=$ false otherwise. For each clause $k \in[m]$, we have exactly one true literal in $a^{\prime}$ because $\left|S^{\prime} \cap P_{k}\right|=1$. So $a^{\prime}$ is a valid assignment for instance $\mathcal{H}$.

Therefore, if $\mathcal{H}$ is unsatisfiable, the optimal value of $\mathcal{E}$ is more than $3-\epsilon$. Theorem 3 now follows.

\section{References}

[CGK20] Deeparnab Chakrabarty, Prachi Goyal, and Ravishankar Krishnaswamy. The nonuniform k-center problem. ACM Transactions on Algorithms (TALG), 16(4):1-19, 2020.

[Che08] Ke Chen. A constant factor approximation algorithm for $k$-median clustering with outliers. In Shang-Hua Teng, editor, Proceedings of the Nineteenth Annual ACMSIAM Symposium on Discrete Algorithms, SODA 2008, San Francisco, California, USA, January 20-22, 2008, pages 826-835. SIAM, 2008.

[CKMN01] Moses Charikar, Samir Khuller, David M Mount, and Giri Narasimhan. Algorithms for facility location problems with outliers. In SODA, volume 1, pages 642-651, 2001.

[CLLW13] Danny Z Chen, Jian Li, Hongyu Liang, and Haitao Wang. Matroid and knapsack center problems. In International Conference on Integer Programming and Combinatorial Optimization, pages 110-122. Springer, 2013. 
[CN19] Deeparnab Chakrabarty and Maryam Negahbani. Generalized center problems with outliers. ACM Transactions on Algorithms (TALG), 15(3):1-14, 2019.

[FG88] Tomás Feder and Daniel Greene. Optimal algorithms for approximate clustering. In Proceedings of the twentieth annual ACM symposium on Theory of computing, pages 434-444, 1988.

[GLS88] Martin Grötschel, Lovász László, and Alexander Schrijver. Geometric Algorithms and Combinatorial Optimization, volume 40. 011988.

[Gon85] Teofilo F Gonzalez. Clustering to minimize the maximum intercluster distance. Theoretical computer science, 38:293-306, 1985.

[HPM04] Sariel Har-Peled and Soham Mazumdar. On coresets for k-means and k-median clustering. In STOC, pages 291-300, 2004.

[HS85] Dorit S Hochbaum and David B Shmoys. A best possible heuristic for the k-center problem. Mathematics of operations research, 10(2):180-184, 1985.

[HS86] Dorit S Hochbaum and David B Shmoys. A unified approach to approximation algorithms for bottleneck problems. Journal of the ACM (JACM), 33(3):533-550, 1986.

[Jai01] Kamal Jain. A factor 2 approximation algorithm for the generalized steiner network problem. Combinatorica, 21(1):39-60, 2001.

$\left[\mathrm{KKN}^{+} 15\right]$ Ravishankar Krishnaswamy, Amit Kumar, Viswanath Nagarajan, Yogish Sabharwal, and Barna Saha. Facility location with matroid or knapsack constraints. Math. Oper. Res., 40(2):446-459, 2015.

[KLS18] Ravishankar Krishnaswamy, Shi Li, and Sai Sandeep. Constant approximation for kmedian and k-means with outliers via iterative rounding. In Ilias Diakonikolas, David Kempe, and Monika Henzinger, editors, Proceedings of the 50th Annual ACM SIGACT Symposium on Theory of Computing, STOC 2018, Los Angeles, CA, USA, June 25-29, 2018, pages 646-659. ACM, 2018.

[KR07] Stavros G. Kolliopoulos and Satish Rao. A nearly linear-time approximation scheme for the Euclidean k-median problem. SIAM J. Comput., 37(3):757-782, 2007.

[NSS20] Viswanath Nagarajan, Baruch Schieber, and Hadas Shachnai. The euclidean $k$-supplier problem. Math. Oper. Res., 45(1):1-14, 2020.

[Ple87] Ján Plesník. A heuristic for the p-center problems in graphs. Discrete Applied Mathematics, 17(3):263-268, 1987.

[Sch78] Thomas J Schaefer. The complexity of satisfiability problems. In Proceedings of the tenth annual ACM symposium on Theory of computing, pages 216-226, 1978.

[Sch03] Alexander Schrijver. Combinatorial optimization: polyhedra and efficiency, volume 24. Springer Science \& Business Media, 2003.

[Swa16] Chaitanya Swamy. Improved approximation algorithms for matroid and knapsack median problems and applications. ACM Trans. Algorithms, 12(4):49:1-49:22, 2016. 